\title{
MHD Flow of a Dusty Viscous Conducting Liquid Between Two Parallel Plates
}

\author{
P. Sreeharireddy ${ }^{*}$, A. S. Nagarajan ${ }^{\#}$ M. Sivaiah* \\ *Department of Mathematics, N.B.K.R. S \& A College, VIdyanagar-524 413 (A.P), India \\ "Department of Mathematics, N.B.K.R.I.S.T, VIdyanagar-524 413 (A.P), India
}

Received 25 October 2008, accepted in final revised form 17 February 2009

\begin{abstract}
In this paper, the flow of a viscous conducting liquid with uniform distribution of dust particles in a channel is considered under the influence of a uniform transverse magnetic field with pressure gradient varying linearly with time. The velocities of fluid and dust are found to decrease with the increase of the magnetic parameter. Further that the velocity of the fluid particles is observed to be more than that of dust particles.

Keywords: Viscous conducting liquid; Uniform transverse magnetic field; Fluidization; Incompressible fluid; Stoke's resistance coefficient.

C 2009 JSR Publications. ISSN: 2070-0237 (Print); 2070-0245 (Online). All rights reserved.

DOI: $10.3329 /$ jsr.v1i2.2280
\end{abstract}

\section{Introduction}

Interest in problems of mechanics of systems with more than one phase has developed rapidly in recent years. The study of fluids having uniform distribution of solid spherical particles is of interest in a wide range of areas of technical importance. These areas include fluidization (flow through packed feds), flow in rocket, tubes, where small carbon or metallic fuel particles are present, environmental pollution, the process by which rain drops are formed by the coalescence of small droplets, which might be considered as solid particles for the purpose of examining their movement prior to coal scene, combustion and more recently blood flow in capillaries. Attia [1] studied unsteady flow of a dusty conducting fluid between parallel porous plates with temperature dependent viscosity. Effect of Hall currents on the MHD flow and heat transfer of a second order fluid between two parallel porous plates was studied by Bhargava and Takhar [2]. Hazem Attia and Kotb [3] studied the MHD flow between two parallel plates with heat transfer. AboulHassan et al. [4] presented a paper on temperature due to motion of one of them between two parallel plates. Reddy [5] studied the flow of dusty viscous liquid through rectangular channel. Work in this field has been carried out by several researchers [6-8]. Saffman [9] investigated the stability of a laminar flow of a dusty gas which is very useful for this

\footnotetext{
${ }^{1}$ Corresponding author: sreeharireddy8969@gmail.com
} 
work. The previous studies done by Bathaiah [10], Sparrow and Cess [11] are much useful in the present study.

In the present investigation, an attempt has been made to study the flow of a dusty viscous and slightly conducting liquid between two parallel flat plates under the influence of a uniform transverse magnetic field. The fluid velocity and the velocity of dust are evaluated and the effect of magnetic field on these velocities is investigated. It is observed that the velocities of fluid and dust decrease with the increase in magnetic parameter $\mathrm{M}$. Further it is noticed that the velocity of the fluid particles is more than that of dust particles.

\section{Formulation and Solution of the Problem}

We consider the flow of a dusty viscous, slightly conducting liquid between two parallel flat plates under the influence of a uniform transverse magnetic field. It is assumed that the fluid is of small electrical conductivity with magnetic Reynolds number much less than unity, so that the induced magnetic field can be neglected in comparison with applied magnetic field.

The equations of motion of a dusty conducting viscous, unsteady and incompressible fluid in the absence of input electric field are [12, 13]:

$$
\begin{gathered}
\frac{\partial \bar{u}}{\partial t}+(\bar{u} \cdot \nabla) \bar{u}=-\frac{1}{\rho} \nabla p+v \nabla^{2} \bar{u}+\frac{K N}{\rho}(\bar{v}-\bar{u})+\frac{\mu_{e}}{\rho}(\bar{J} \times \bar{H}) \\
m\left[\frac{\partial \bar{v}}{\partial t}+(\bar{v} \cdot \nabla) \bar{v}\right]=K[\bar{u}-\bar{v}] \\
\operatorname{div} \bar{u}=0 \\
\frac{\partial N}{\partial t}+\operatorname{div}(N \bar{v})=0
\end{gathered}
$$

where $\bar{u}$ and $\bar{v}$ are velocities of fluid and dust particles respectively; $t$, the time, $p$, the fluid pressure; $\rho$, the fluid density; $v$, the kinematic coefficient of viscosity; $k$, the Stokes's resistance coefficient which for spherical particle of radius $\mathrm{r}$ is $6 \pi \mu \mathrm{r}$. $N$ represents the number density of the dust particles, $\mu_{e}$ the magnetic permeability, $J$ the current density, $H$ the magnetic field and $m$ the mass of the dust particles.

The $\mathrm{x}$-axis is taken along the mid way of the channel and a straight line perpendicular to that is taken as the y-axis. The magnetic field of intensity $H_{0}$ is introduced in the ydirection.

For the present problem the velocity distribution of fluid and dust particles are defined respectively as

$$
u_{1}=u_{1}(y, t), \quad u_{2}=0, \quad u_{3}=0
$$




$$
v_{l}=v_{1}(y, t), \quad v_{2}=0, \quad v_{3}=0
$$

where $\left(u_{1}, u_{2}, u_{3}\right)$ and $\left(v_{1}, v_{2}, v_{3}\right)$ are the velocity fields of fluid and dust, respectively.

Now using these, Eqs. (1) and (2) become

$$
\begin{gathered}
\frac{\partial u_{1}}{\partial t}=-\frac{1}{\rho} \frac{\partial p}{\partial x}+v \frac{\partial^{2} u_{1}}{\partial y^{2}}+\frac{l}{\tau}\left(v_{1}-u_{1}\right)-\frac{\sigma \mu_{e}^{2} H_{0}^{2}}{\rho} u_{1} \\
\frac{\partial v_{1}}{\partial t}=\frac{1}{\tau}\left(u_{1}-v_{1}\right)
\end{gathered}
$$

where $\quad l=\frac{m N}{\rho}$ (mass concentration), and $\tau=\frac{m}{K}$ (time relaxation).

The boundary conditions are

$$
\left.\begin{array}{lll}
u_{1}=0, v_{l}=0 & \text { at } & y=-h \\
u_{l}=0, v_{l}=0 & \text { at } & y=+h
\end{array}\right\}
$$

We introduce non-dimensional quantities $t^{l}, u_{1}{ }^{1}, v_{l}{ }^{l}, x^{l}, y^{l}, p^{l}$ and $z^{l}$, As follows

$$
\begin{array}{ll}
t^{1}=\frac{v t}{h^{2}}, \quad u_{1}^{1}=\frac{u_{1}}{u_{0}}, & v_{1}^{1}=\frac{v_{1}}{u_{0}}, \quad x^{1}=\frac{x}{h}, \\
y^{1}=\frac{y}{h}, P^{1}=\frac{P}{\rho u_{0}^{2}}, & \tau^{1}=\frac{\tau v}{h^{2}}=\frac{m v}{K h^{2}}
\end{array}
$$

where $u_{0}$ is the characteristic velocity and $h$ the half distance between the plates.

In view of Eq. (9), Eqs. (6) and (7) reduce to (dropping primes).

$$
\begin{aligned}
\frac{\partial u_{1}}{\partial t} & =-R \frac{\partial p}{\partial x}+\frac{\partial^{2} u_{1}}{\partial y^{2}}+\frac{l}{\tau}\left(v_{1}-u_{1}\right)-M u_{1} \\
\frac{\partial v_{1}}{\partial t} & =\frac{1}{\tau}\left(u_{1}-v_{1}\right)
\end{aligned}
$$

where $R=\frac{\mu_{0} h}{v}$ (Reynolds number) and $M=\frac{\sigma \mu_{e}^{2} H_{0}^{2} h^{2}}{\mu}$ (magnetic parameter)

Eliminating $v_{l}$ from the Eq. (10) and (11), we obtain

$$
\frac{\partial^{2} u_{1}}{\partial t^{2}}+\frac{\partial}{\partial t}\left(R \frac{\partial p}{\partial x}\right)-\frac{\partial}{\partial t}\left(\frac{\partial^{2} u_{1}}{\partial y^{2}}\right)+\left[\frac{l+1}{\tau}+M\right] \frac{\partial u_{1}}{\partial t}+\frac{1}{\tau}\left[R \frac{\partial p}{\partial x}-\frac{\partial^{2} u_{1}}{\partial y^{2}}\right]=0
$$

The boundary conditions in the non-dimensional form are

$$
\mathrm{u}_{1}=0, \mathrm{v}_{1}=0 \quad \text { at } \quad \mathrm{y}=-1
$$




$$
\mathrm{u}_{1}=0, \mathrm{v}_{1}=0 \quad \text { at } \quad \mathrm{y}=1
$$

Suppose that

$$
-R \frac{\partial p}{\partial x}=a_{0}+a t
$$

and

$$
u_{1}=f(y)\left(a_{0}+a t\right)+a g(y)
$$

where $f$ and $g$ are functions of $y$ only.

In view of Eqs. (14) and (15), Eq. (12) reduces to

$$
-a\left[1+f^{11}(y)-\left\{\frac{l+1}{\tau}+M\right\} f(y)-\frac{g^{11}(y)}{\tau}\right]-\frac{a_{0}+a t}{\tau}\left[1+f^{11}(y)\right]=0
$$

where primes denote differentiation with respect to $y$ we can obtain the expressions for $f(y)$ and $g(y)$ from Eq. (16) by equating the coefficients of $\left(a_{0}+a_{t}\right)$ and a to zero

Thus

$$
\begin{gathered}
f(y)=\frac{1}{2}\left(1-y^{2}\right) \\
g(y)=\frac{\tau}{24}\left[\frac{l+1}{\tau}+M\right]\left(6 y^{2}-y^{4}-5\right)
\end{gathered}
$$

Then from Eqs. (15), (17) and (18), we obtain the velocity of the fluid

$$
u_{1}=\frac{1}{2}\left(a_{0}+a t\right)\left(1-y^{2}\right)+\frac{a \tau}{24}\left\{\frac{l+1}{\tau}+M\right\}\left(6 y^{2}-y^{4}-5\right)
$$

From Eqs. (11) and (19) we get the velocity of the dust

$$
v_{1}=\frac{\tau}{l}\left[\frac{1}{2} a\left(1-y^{2}\right)-\frac{a \tau}{2}\left\{\frac{l+1}{\tau}+M\right\}\left(1-y^{2}\right)+\left(\frac{l}{\tau}+M\right) u_{1}\right]
$$

\section{Results and Discussions}

The results obtained on the velocity of the fluid particles $\left(u_{l}\right)$ and dust particles $\left(v_{l}\right)$ due to the variation of the parameters $l, \tau$ and $t$ under the influence of the magnetic field are shown in Figs. 1-4. It is noticed that variation in magnetic parameter $(M)$ causes significant changes in the velocity of fluid particles as well as in the velocity of dust particles. It is observed that the velocity of both the fluid and dust particles decreases as the magnetic parameter $M$ increases. But a close look at Fig.1 reveals that the effect of magnetic parameter $M$ is slight on the velocity of the dust particles when compared to the fluid particles. This result follows the logic that the velocity of the fluid particles is more than that of the dust particles. 

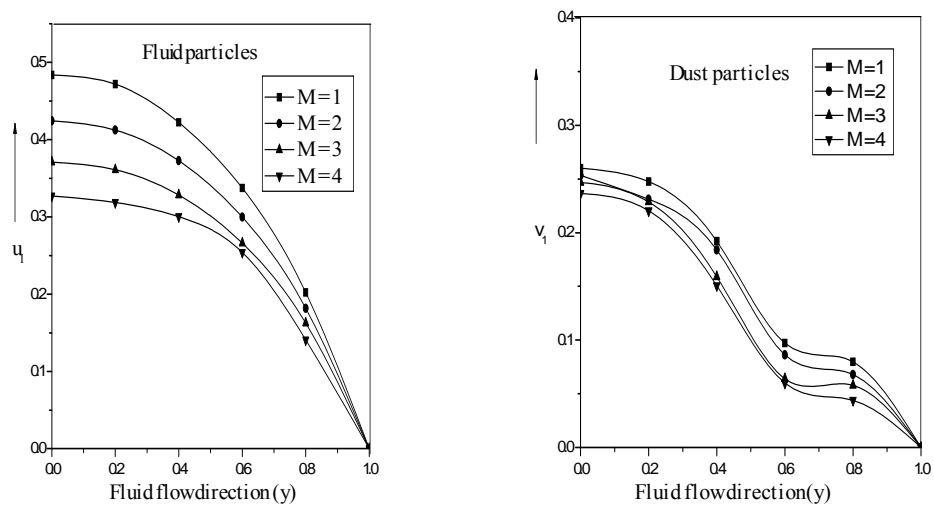

Fig. 1. Velocity profile for different values of $M$ for (a) fluid particles and (b) dust particles.
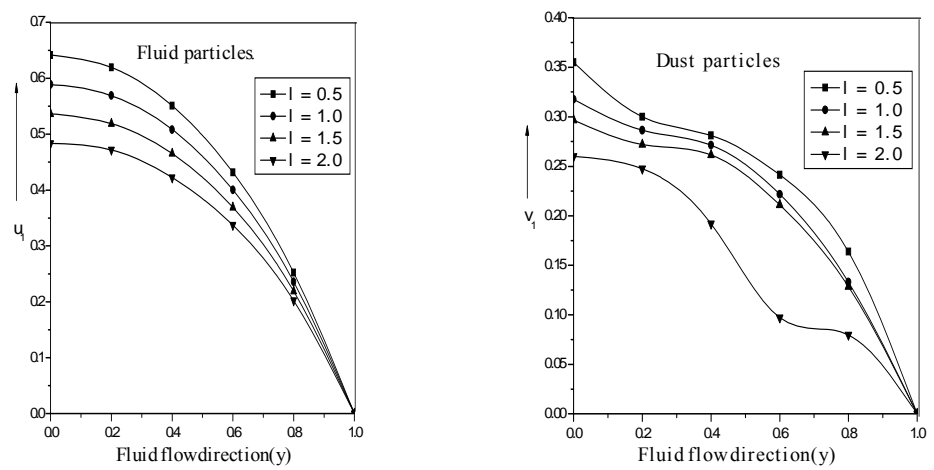

Fig. 2. Velocity profiles for different values of mass concentration $l$ for (a) fluid particles and (b) dust particles.
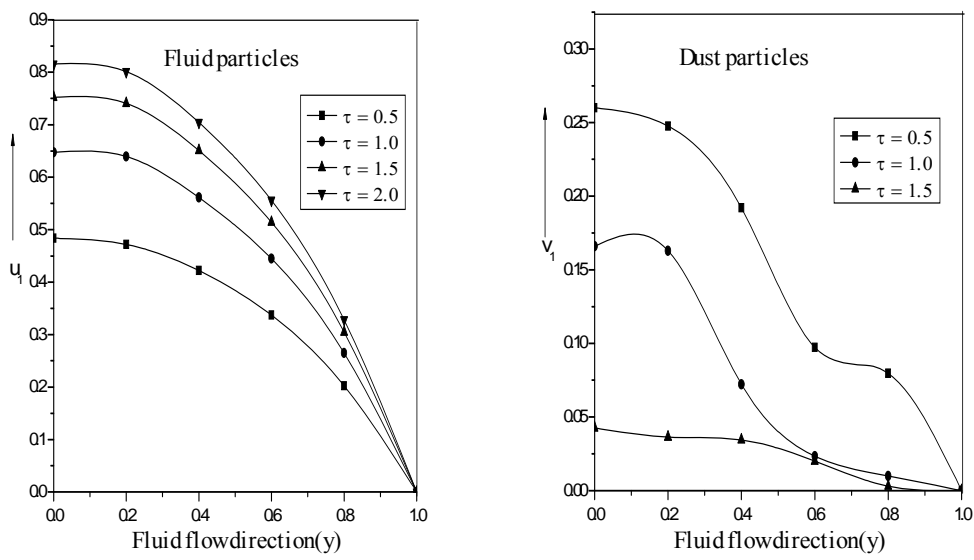

Fig. 3. Velocity profile for different values of time relaxation $\tau$ for (a) fluid particles and (b) dust particles. 
Fig. 2 illustrates the effect of mass concentration $l$ on the velocity of the fluid and dust particles. It is noticed that velocity of the fluid and dust particles decreases with the increasing values of mass concentration $l$. This is in agreement with the fact that the mass of the dust particles is small, then the influence of the fluid flow is reduced and as $m \rightarrow 0$, the fluid becomes ordinary viscous.
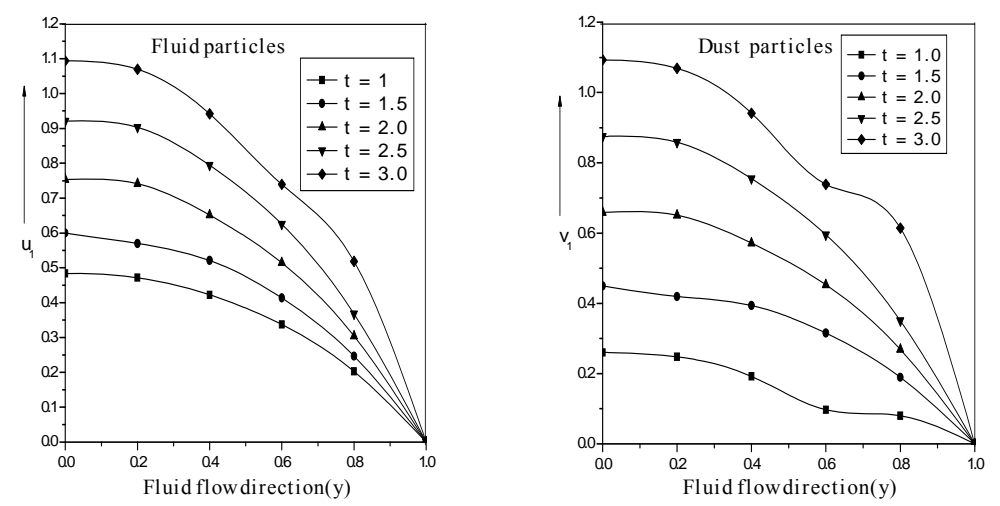

Fig. 4. Velocity profile for different values of time $t$ for (a) fluid particles and (b) dust particles.

The effect of time relaxation $\tau$ on the velocities of fluid and dust particles is shown in Fig. 3. It is observed that the velocity of fluid particles increases with increase in $\tau$, whereas in case of dust particles the trend is reversed and the dust particle velocity tends to zero as $\tau$ increases. If the dust is very fine, i.e. mass of the dust particles is negligibly small, then the relaxation time of dust particles decreases and ultimately as $\tau \rightarrow 0$ the velocities of fluid and dust particles will be the same. Finally in case of time $t$ as shown in Fig. 4, we observe that the velocity of both fluid and dust particles increases with the increase in time. For $t>3$, considerable difference is not observed in the velocity of fluid and dust particles.

\section{References}

1. A. Hazem Attia, Turk J. Phys. 29, 257 (2005).

2. R. Bhargava and H. S. Takhar, J. MHD, Plasma \& space research 10, 73 (2001).

3. A. Hazem Attia and N. A. Kotb, Acta Mechanica 117, 215 (1996). doi:10.1007/BF01181049

4. A. L. Aboul-Hassan, H. Sharaf El-Din and A. L. Megahed, First International Conference of Engineering Mathematics and Physics, Cairo (1991) p. 723.

5. Y. B. Reddy, Def. Sci. J. 22, 149 (1972).

6. D. H. Michael, Proc. Camb. Phil. Soc. 61, 569 (1965). doi:10.1017/S030500410000414X

7. P. S. S. Rao, Def. Sci. J. 19, 135 (1969).

8. P. D. Varma and A.K. Mathur, Ind. J. Pure \& Appl. Math. 4, 133 (1973).

9. P. G. Saffman, J. Fluid Mech. 13, 120 (1962). doi:10.1017/S0022112062000555

10. D. Bathaiah, Acta Mechanica 35, 223 (1980). doi:10.1007/BF01190398

11. E. M. Sparrow and R. D. Cess, J. Appl. Mech. 29, 181 (1962).

12. R. Venugopal and D. Bathaiah, Def. Sci. J. 33 (1), 69 (1983).

13. G. Radhakrishnama Charya, ZAMP, 29, 217 (1978). 